\title{
Rectovaginal septum as a site for recurrent myofibroblatic tumor (first time report)
}

\section{Background}

Myofibroblastic tumor (MT) is a neoplasm of unknown etiology, occurring at various sites but was not reported in the rectovaginal area. Literary it is composed of spindle cells (myofibroblasts). Usually it is associated with variable inflammatory component; hence the name is inflammatory myoblastic tumor (IMT). The occurrence in the rectovaginal septum of female is almost unknown in the literature.

\begin{abstract}
Otherwise healthy 35 year old female, 6 months postpartum, presented with a vaginal mass and difficult intercourse. Gynecologic examination demonstrated a normal uterus with a posterior vaginal wall $10 \mathrm{~cm}$ rounded mass. Excision and pathology proved the diagnosis of myofibrobalstic tumor.
\end{abstract}

Conclusion: Myofibroblastic tumor although very rare, should be considered in the differential diagnosis of masses in the posterior vaginal wall, rectovaginal septum or pouch of Douglas.

Keywords: tumor, septum, inflammatory, vaginal

\author{
Volume 2 Issue 4 - 2015
}

Galal Lotfi

Suez Canal University, Egypt

Correspondence: Galal Lotfi, 14A Sherif Street, Roxy, Cairo I I34I, Egypt, Tel 20-2-24535597, Email glotfi@gmail.com

Received: November 18, 20I4 | Published: July 15, 2015

\section{Abbreviations}

MT, myofibroblastic tumor; IMT, inflammatory myoblastic tumor; IPT, inflammatory pseudotumor tumor; EBV, epstein barr virus; HHV8, human herpes virus; ORF, open reading frame.

\section{Background}

Myofibroblastic tumor is usually associated with some inflammatory cells, hence named inflammatory Myofibroblastic tumor (IMT) and sometimes known as inflammatory pseudotumor tumor (IPT). It is a lesion of unknown etiology that has been reported in numerous anatomic sites. The tumor is composed of a dominant spindle cell proliferation with a variable inflammatory component. These spindle cells are known to be myofibroblasts and this is the reason for the current designation for this disease. Inflammatory factor may not be applicable to all tumors, since some investigators have demonstrated the presence of chromosomal abnormalities and documented cases showing aggressive behavior supporting the theory that at least some of these tumors are true neoplasms. The tumor is more common in children with no predilection for sex but in adult age it is more common in women, who often present with fever of unknown origin or other vague, nonspecific symptoms. Splenomegaly is a frequent finding. The tumor is almost unknown to the recto signal septum. It was reported inretroperitoneal region, ${ }^{1-3}$ gastrointestinal tract, breast, ${ }^{4}$ lung, ${ }^{1}$ heart, urinary tract, ${ }^{5}$ uterus, pancreas. ${ }^{6}$ The outlook of this disease has changed with time from a benign reactive process to a malignant neoplasm, based on the multiple case reports demonstrating recurrent and constant clonal genetic alterations. ${ }^{7-10}$

IMT is a spindle cell proliferation that can histologically resemble various malignant mesenchymal neoplasms; however, it generally behaves as a benign or locally recurrent tumor.

\section{Case report}

MS is a 35 year old $\mathrm{P} 1+0+0+1$ who had cesarean section 6 months earlier. She presented with vaginal mass that was painless but causing difficult intercourse. She had been interviewed by general surgeon who found $8-10 \mathrm{~cm}$ mass related to the posterior vaginal wall. He had performed a laparotomy, trying to reach the mass through the pouch of Douglas and when he failed, he tried to have a biopsy which was insufficient and inconclusive. 3 weeks later she reported to our department, where examination showed an $8-10 \mathrm{~cm}$ sized mass between the rectum and posterior vaginal wall slightly shifted to the right side of the midline. The mass was not cystic but firm, mobile and not tender and extending from $2 \mathrm{~cm}$ above the introitus up to the level of posterior vaginal fornix. Rectal examination showed that the mass was in front of the rectum and upper part of the anal canal. A CT (Figure 1) and directed biopsy (Figure 2), was carried out and it showed $8-10 \mathrm{~cm}$ mass abutting the rectum but not infiltrating; the biopsy showed fibrous nature. Apart from that the patient gave no history of anything important and the examinations of all other systems were negative. She denied fever, her postpartum course had been uncomplicated and she was exclusively breast feeding since delivery. Laboratory data were unremarkable.

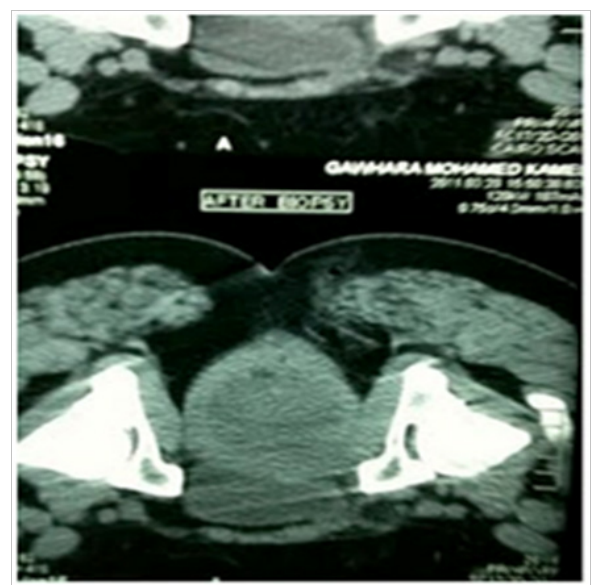

Figure I CT Image. 
Vaginal route exploration for excision of the mass was arranged. The posterior vaginal wall was dissected off the mass upwards the same way of doing rectocele repair. The mass was having a false capsule and this helped in its dissection from the posterior vaginal wall. To dissect the mass from the rectum and the anal canal, the assistant inserted his finger in the anal canal trying to push the mass forward and anteriorly to put it under tension to help in its dissection and in the meanwhile alarming if dissection went close to the rectum to avoid its injury (Figures $3 \& 4$ ). Dissection continued and succeeded in taking the mass out (Figure 5) and it was extending from the introitus up to the pouch of Douglas. Closure was carried out, a drain was left that was removed after 24 hours and the postoperative period passed uneventful.

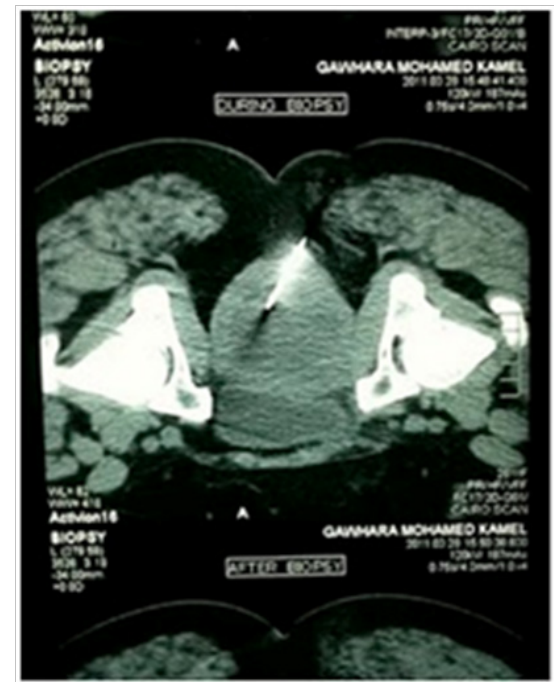

Figure 2 CT directed biopsy.

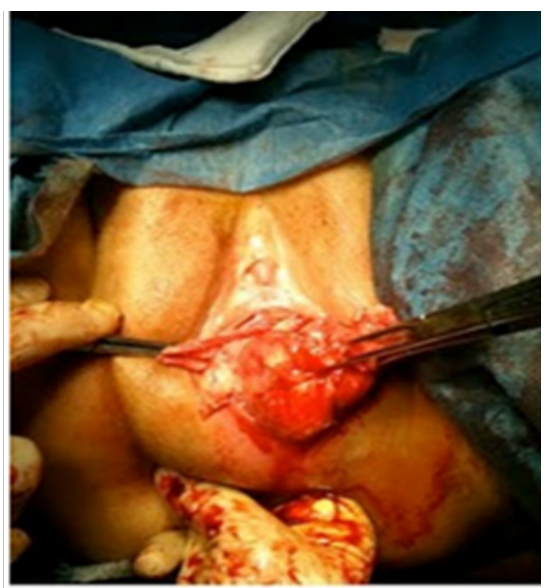

Figure 3 Dissection of the tumor (assisting inserting finger in a anal canal).

The patient had an uncomplicated recovery and was discharged home. The pathology report came back with a diagnosis of IMT, benign in nature. Six months later, the patient presented herself for follow up with a main complaint of dyspareunia, CT was carried out and it showed the appearance of a mass of $6 \mathrm{~cm}$ in the middle portion of rectovaginal septum. Vaginal rout dissection and excision was carried out. Postoperative period was uneventful and the pathology report was the same. The patient was reviewed after 6 months, symptom free and
CT scan was free of any lesions. 18 months after the last operation, the patient got pregnant and elective repeat cesarean section was carried out, the outcome was a live baby boy $3200 \mathrm{Gm}$, and postoperative period passed uneventful.

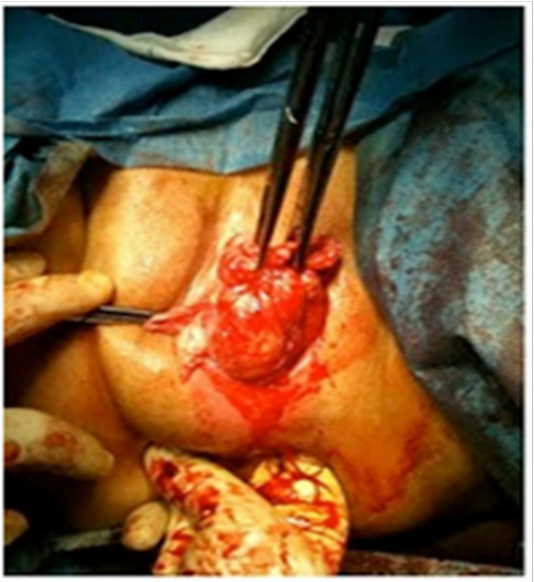

Figure 4 Dissection.

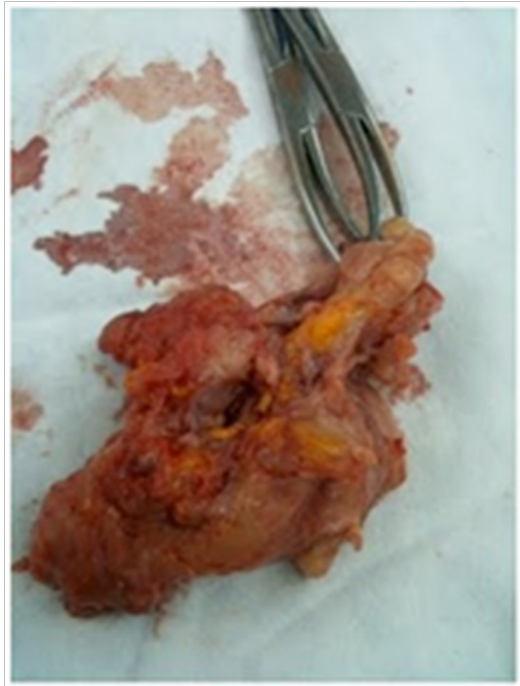

Figure 5 Gross picture of tumor.

\section{Discussion}

IMT is a relatively rare enigma that is almost unknown to the gynaecologist. Sometimes it is referred to as plasma cell granuloma, or inflammatory pseudo tumor (IPT). It has long been debated regarding the origin of IMT whether it was truly neoplastic or a post inflammatory process. The proposed etiologies included Epstein Barr virus (EBV), Human herpes virus (HHV8), and over expression of interleukin IL-6. Though other diseases like Kaposi's sarcoma and Castleman's diseases also have similar etiologies, molecular transcription form of open reading frame (ORF)-16, K13, 72 expressed in IMT are not expressed in the aforesaid diseases. ${ }^{11}$ Recently IMT is considered a neoplasm rather than a post-inflammatory process because of cytogenetic clonality, recurrent involvement of chromosomal region, occasional aggressive local behavior and metastasis of the tumor. ${ }^{1}$ With a differential diagnosis of amass in the rectovaginal septum, one should always consider endometriotic nodule, tuberculosis or 
metastases, as it is mentioned an all the teaching text books. Of course that triad was considered, and was ruled out in our case by ultrasound, CT directed biopsy, and the past medical history. The mobility of the mass and rectal examination almost excluded a malignant cause either rectal or metastatic origin. Endometriotic nodule could be excluded by clinical history of pain and abnormal uterine bleeding in addition to rectal symptoms like tenesmus and constipation.

Excision was the definitive treatment; however, the route of the excision was a matter of debate. The general surgeon when asked preferred the abdominal exploration but since gynecologists know that anatomical area more than any other specialty physician, the vaginal route was opted. Recurrence of the lesion is common and prolonged follow up is a necessity. ${ }^{12}$

\section{Conclusion}

IMT is a rare an enigma of uncertain biological potential. It is unknown to the gynaecologist as far as the rectovaginal septum is concerned. Complete surgical resection remains the mainstay of the treatment. IMT although rare, should be considered as a diagnosis. Recurrence should be expected.

\section{Acknowledgments}

None.

\section{Conflicts of interest}

The authors declare there is no conflict of interests.

\section{References}

1. Romero R, Espinoza J, Gonçalves LF, et al. The role of inflammation and infection in preterm birth. Semin Reprod Med. 2007;25(1):21-39.

2. Coffin CM, Watterson J, Priest JR, et al. Extrapulmonary inflammatory myofibroblastic tumor (inflammatory pseudotumor). A clinic pathologic and immuno-histochemical study of 84 cases. Am J Surg Pathol. 1995;19(8):859-872.
3. Esmer-Sanchez D, Rangel D. Inflammatory pseudotumor of the retroperitoneum. Rev Gastroenterol Mex. 2002;67(2):97-99.

4. Tambo M, Kondo H, Kitauchi $\mathrm{T}$, et al. A case of inflammatory myofibroblastic tumor of the retroperitoneum. Hinyokika Kiyo. 2003;49(5):273-276.

5. Sastre-Garau X, Couturier J, Derré J, et al. Inflammatory myofibroblastic tumour (inflammatory pseudotumour) of the breast. Clinicopathological and genetic analysis of a case with evidence for clonality. J Pathol. 2002;196(1):97-102.

6. Kapusta LR, Weiss MA, Ramsay J, et al. Inflammatory myofibroblastic tumors of the kidney: a clinicopathologic and immunohistochemical study of 12 cases. Am J Surg Pathol. 2003;27(5):658-666.

7. Pungpapong S, Geiger XJ, Raimondo M. Inflammatory myofibroblastic tumor presenting as a pancreatic mass: a case report and review of the literature. JOP. 2004;5(5):360-367.

8. Coffin CM, Dehner LP, Meis-Kindblom JM. Inflammatory myofibroblastic tumor, inflammatory fibrosarcoma, and related lesions: an historical review with differential diagnostic considerations. Semin Diagn Pathol. 1998;15(12):102-110.

9. Freeman A, Geddes N, Munson P, et al. Anaplastic lymphoma kinase (ALK 1) staining and molecular analysis in inflammatory myofibroblastic tumors of the bladder: a preliminary clinicopathological study of nine cases and review of the literature. Mod Pathol. 2004;17(7):765-771.

10. Biselli R, Boldrini R, Ferlini C, et al. Myofibroblastic tumors: neoplasias with divergent behavior. Ultrastructural and flow cytometric analysis. Pathol Res Pract. 1999;195(9):619-632.

11. Mentzel T, Dry S, Katenkamp D, et al. Low-grade myofibroblastic sarcoma: analysis of 18 cases in the spectrum of myofibroblastic tumors. Am J Surg Pathol. 1998;229(10):1228-1238.

12. Gomez-Roman JJ, Sanchez-Velasco P, Ocejo-Vinyals G, et al. Human herpesvirus-8 genes are expressed in pulmonary inflammatory myofibroblastic tumor (inflammatory pseudotumor). Am J Surg Pathol. 2001;25(5):624-629. 